\title{
EDITORIAL
}

\section{Beta-blockers in septic shock to optimize hemodynamics? No}

\author{
Anthony S. McLean ${ }^{1 *}$, Fabio S. Taccone ${ }^{2}$ and Antoine Vieillard-Baron ${ }^{3,4}$
}

๑ 2016 Springer-Verlag Berlin Heidelberg and ESICM

Cardiac assessment in the critically ill septic patient has become increasingly sophisticated [1]; yet, the relationship between the heart and systemic arterial system remains an area of unfulfilled hope, with precise evaluation continuing to elude the practicing clinician. The concept of "cardiovascular (CV) performance or efficiency" is an attractive one, a tool promising to bridge the knowledge gulf that currently exists [2]. That said, its clinical application is challenging. First, there seems to be a lack of consensus as to which parameters best represent $\mathrm{CV}$ performance, whether it be stroke volume (SV), stroke work (SW), work efficiency, or the ventriculo arterial coupling (V-A coupling) [3]. Second, pragmatic methods for measuring some theoretical parameters, such as unstressed ventricular volume $\left(V_{0}\right)$, end-systolic pressure $\left(P_{\mathrm{es}}\right)$, and arterial compliance $\left(C_{\mathrm{art}}\right)$, are still lacking, let alone the challenge of the time-variable nature of these parameters.

Over a quarter of a century ago, in the effort to match the heart function and the arterial load from an evolutionary viewpoint, Elzinga and Westerhof postulated that to attain minimum ventricular size a mammalian heart evolved with its working point quite close to optimum power and optimum efficient, and that a specific heart rate is required to guarantee sufficient pressure during diastole [4]. A decade prior to this, Sunagawa et al. predicted that maximal SW results when the end-systolic elastance $\left(E_{\mathrm{es}}\right)$ of the ventricle and arterial load $\left(E_{\mathrm{a}}\right.$, effective arterial elastance) are equal $E_{\mathrm{a}} / E_{\mathrm{es}}=1[5,6]$.

The $E_{\mathrm{a}} / E_{\text {es }}$ ratio has been used in various studies as a means to reflect V-A coupling $[2,7,8]$. In these studies,

\footnotetext{
${ }^{*}$ Correspondence: anthony.mclean@sydney.edu.au

1 Department of Intensive Care Medicine, Nepean Hospital, University of Sydney, Sydney, NSW, Australia

Full author information is available at the end of the article
}

For contrasting viewpoints, please go to doi:10.1007/s00134-016-4414-4 and doi:10.1007/s00134-016-4421-5. a high $E_{\mathrm{a}} / E_{\mathrm{es}}$ ratio is taken to imply $\mathrm{V}$-A uncoupling. $E_{\mathrm{a}}$ has a determinative effect on V-A coupling if ventricular contractility, hence $E_{\mathrm{es}}$, is constant. On the basis of the pressure-volume relationship, Sunagawa et al. predicted, using the ratio of left ventricular $P_{\mathrm{es}}$ to $\mathrm{SV}$, that $E_{\mathrm{a}}$ remains constant under a given steady-state vascular impedance load [5]. Hence, this gives rise to

$$
E_{\mathrm{a}}=\frac{P_{\mathrm{es}}}{\mathrm{SV}}
$$

Although such measurements originally required invasive pressure recordings, now hemodynamic monitoring techniques allow for estimation of $E_{\mathrm{a}}$ by measuring SV noninvasively. $E_{\mathrm{a}}$ can be expressed as a function of systemic vascular resistance (SVR), heart rate (HR), and $C_{\text {art }} P_{\text {es }}$ is higher than mean arterial pressure (MAP) in humans, and it is expressed as follows:

$$
P_{\mathrm{es}}=\mathrm{MAP}+\Delta P
$$

As MAP is the product of SVR and cardiac output (CO), it follows that

$$
\begin{aligned}
& P_{\mathrm{es}}=(\mathrm{SVR} \times \mathrm{CO})+\Delta P \\
& P_{\mathrm{es}}=(\mathrm{SVR} \times \mathrm{SV} \times \mathrm{HR})+\Delta P \\
& \frac{P_{\mathrm{es}}}{\mathrm{SV}}=(\mathrm{SVR} \times \mathrm{HR})+\frac{\Delta P}{\mathrm{SV}}
\end{aligned}
$$

Since $P_{\mathrm{es}} / \mathrm{SV}$ is $E_{\mathrm{a}}$, and $\Delta P / \mathrm{SV}$ is a measure of arterial stiffness $\left(1 / C_{\text {art }}\right)$, hence

$$
E_{\mathrm{a}}=(\mathrm{SVR} \times \mathrm{HR})+\frac{1}{C_{\mathrm{art}}}
$$

Hence, $E_{\mathrm{a}}$ can be approximated as MAP/SV $+1 / C_{\text {art }}$. The above equations state that $E_{\mathrm{a}}$ consists of two components: a steady component (SVR $\times$ HR) and a pulsatile component $\left(1 / C_{\mathrm{art}}\right)$ [7]. Using regression analysis,

\section{实


Segers et al. not only confirmed the above relationship in a heart-arterial interaction model but also found that the first component contributes three times more to $E_{\mathrm{a}}$ than the second [9], such difference probably being lower in septic patients with vasoplegia.

Morelli et al. recently suggested that the short-acting $\beta$-blocker esmolol improves CV efficiency in selected septic patients, e.g., those who remained tachycardic after initial resuscitation and still requiring norepinephrine infusion [10]. They hypothesized that these findings could partially explain the better prognosis associated with such therapy observed in a previous study targeting the same population [11]. Unfortunately, Morelli and colleagues may have failed to make the leap across the gap of understanding the $\mathrm{CV}$ efficiency in their study on several fronts.

First, there is no agreement for the choice of best parameters to reflect $\mathrm{CV}$ efficiency and that is very much left to investigators [3]. Morelli's group evaluated $E_{\mathrm{a}}$ [10], as an indirect marker of $E_{\mathrm{a}} / E_{\mathrm{es}}$ ratio, assuming that $E_{\mathrm{es}}$ remains constant [10]. They understood the decrease in $E_{\mathrm{a}}$ observed after esmolol infusion as a better CV efficiency, leading to an increase in SV. However, $E_{\mathrm{a}} / E_{\mathrm{es}}$ does not correlate linearly with SW and CV efficiency which have been reported to decrease with increasing or decreasing $E_{\mathrm{a}} / E_{\mathrm{es}}$ ratio [12]. Optimal SW and CV efficiency have been reported with an $E_{\mathrm{a}} / E_{\text {es }}$ of 1 and between 0.5 and 0.66 , respectively [12].

Second, most investigators used the dicrotic notch or $90 \%$ of peak systolic pressure $\left(P_{\text {dic }}\right)$ in their calculations, and Morelli's estimation of $E_{\mathrm{a}}$ is based on MAP, calculating the ratio between MAP and SV. Not only does this lead to an underestimation of $E_{\mathrm{a}}$ but also arterial stiffness $\left(1 / C_{\text {art }}\right)$ is excluded from the calculations. That said, they also reported the difference between MAP and $P_{\text {dic }}$ using the MostCare ${ }^{\circledR}$ hemodynamic monitor. Whereas this difference is negligible in healthy subjects, this is higher in septic patients as a result of decrease in vascular tone and was partially restored after esmolol in Morelli's study [10].

Third, confounders altering SVR, HR, vascular tone, preload, and contractility can all affect the estimations and interpretations of $E_{\mathrm{a}}$ and $E_{\mathrm{es}}$. Like most other sepsis studies, Morelli's study was 'contaminated' with such confounders: interferences of catecholamines on SVR and increasing ventricular preload, the variation of underlying effects of sepsis on both the arterial tree as well as the left ventricle, and concomitant changes in ventricular elastance $\left(E_{\mathrm{es}}\right)$ that may possibly match changes in the measured $E_{\mathrm{a}}$, maintaining V-A coupling within an acceptable range. For example it is known that with changing heart rates in normal subjects any changes in $E_{\mathrm{a}}$ are matched by corresponding changes in $E_{\mathrm{es}}$, maintaining V-A coupling around 1.
Finally, their interesting assumption is that with no change in left ventricular ejection fraction (LVEF) or $\mathrm{CO}, E_{\mathrm{a}}$ reduction is responsible for the increase in SV. Yet for an increase in SV there must also be a larger left ventricular end-diastolic volume for LVEF to remain the same. As a consequence, LV wall-stress would increase, then limiting improvement in $\mathrm{CV}$ performance. And $\mathrm{HR}$ has been shown to influence both end-diastolic and endsystolic volume [13]. Unfortunately, Morelli et al. did not report changes in LV size. Hence, there must be some change in ventricular function, even if it is an increase in preload that contributes to the results. Indeed, it is very difficult to change loading conditions without provoking reflex changes in ventricular contractility.

The range of hemodynamic measuring devices, including pulmonary artery catheter, thermodilution $\mathrm{CO}$ measuring devices, peripheral arterial pulse contour analysis, and echocardiography, attest to the enthusiasm of Morelli and colleagues [10]. However, their conclusions in regard to arterial elastance changes in septic patients when using esmolol raise too many uncertainties to be confident of the conclusions. Moreover, assuming that $\beta$-blockers could be useful in septic shock, and because of their negative inotropic effect, it is crucial to better understand in which patients such a drug could be efficient and not dangerous. In this regards, echocardiography should be key by its ability to detect severe septic cardiomyopathy (contraindication or non-indication to beta-blockers?) or hyperkinetic left ventricle (theoretical population of interest?) [14], which was not the case in the papers by Morelli's group $[10,11]$.

In conclusion, while the theoretical background is firmly laid, translating these concepts into practical use still requires lots of research since all the approximations and omissions previously discussed elevate doubts about the meaning of any results obtained and conclusions drawn. It is only fair to point out that such challenges bedevil most researchers in this field.

\section{Author details \\ ${ }^{1}$ Department of Intensive Care Medicine, Nepean Hospital, University of Syd- ney, Sydney, NSW, Australia. ${ }^{2}$ Department of Intensive Care, Hôpital Erasme, Université Libre de Bruxelles, Brussels, Belgium. ${ }^{3}$ Medical-Surgical Intensive Care Unit, University Hospital Ambroise Paré, Assistance Publique-Hôpitaux de Paris, Boulogne-Billancourt, France. ${ }^{4}$ INSERM U-1018, CESP, Team 5, University of Versailles Saint-Quentin en Yvelines, Villejuif, France.}

\section{Compliance with ethical standards}

\section{Conflicts of interest}

Anthony McLean declares no conflict of interest. Fabio Silvio Taccone declares no conflict of interest. Antoine Vieillard-Baron declares no conflict of interest.

Received: 4 May 2016 Accepted: 25 May 2016

Published online: 27 June 2016 


\section{References}

1. Chang WT, Lee WT, Chen PS, Su YR, Liu PY, Liu YW, Tsai WC (2015) Left ventricular global longitudinal strain is independently associated with mortality in septic shock patients. Intensive Care Med 41:1791-1799. doi:10.1007/s00134-015-3970-3

2. Guarracino F, Ferro B, Morelli A, Bertini P, Baldassarri R, Pinsky MR (2014) Ventriculo-arterial decoupling in human septic shock. Crit Care 18:R80

3. Starling MR (1993) Left ventricular-arterial coupling relations in the normal human heart. Am Heart J 125:1659-1666

4. Elzinga G, Westerhof N (1991) Matching between ventricle and arterial load. An evolutionary process. Circ Res 68:1495-1500

5. Sunagawa K, Maughan WL, Burkhoff D, Sagawa K (1983) Left ventricular interaction with arterial load studied in isolated canine ventricle. Am J Physiol 245:H773-H780

6. Sunagawa K, Maughan WL, Sagawa K (1985) Optimal arterial resistance for the maximal stroke work studied in isolated canine left ventricle. Circ Res 56:586-595

7. Cohen-Solal A, Caviezel B, Himbert D, Gourgon R (1994) Left ventriculararterial coupling in systemic hypertension: analysis by means of arterial effective and left ventricular elastances. J Hypertens 12:591-600

8. Chantler PD, Lakatta EG (2012) Arterial-ventricular coupling with aging and disease. Front Physiol 3:1-12
9. Segers P, Stergiopulos N, Westerhof N (2002) Relation of effective arterial elastance to arterial system properties. Am J Physiol Heart Circ Physio 282:H1041-H1046

10. Morelli A, Singer M, Ranieri VM, D'Egidio A, Mascia L, Orecchioni A et al (2016) Heart rate reduction with esmolol is associated with improved arterial elastance in patients with septic shock: a prospective observational study. Intensive Care Med. doi:10.1007/s00134-016-4351-2

11. Morelli A, Ertmer C, Westphal M, Rehberg S, Kampmeier T, Ligges S et al (2013) Effect of heart rate control with esmolol on hemodynamic and clinical outcomes in patients with septic shock. JAMA 310:1683-1691

12. Kass DA, Kelly RP (1992) Ventriculo-arterial coupling: concepts, assumptions, and applications. Ann Biomed Eng 20:41-62

13. Ricci DR, Orlick AE, Alderman EL, Alderman EL, Ingels NB Jr, Daughters GT 2nd, Stinson EB (1979) Influence of heart rate on left ventricular ejection fraction in human beings. Am J Cardiol 44:447-451

14. Vieillard-Baron A, Cecconi M (2014) Understanding cardiac failure in sepsis. Intensive Care Med 40:1560-1563 